Original Research Article

\title{
Acquisition of prescribing skill by medical students in a problem-based learning curriculum: preclerkship to clerkship phase transition
}

\author{
Khalid A. J. Al Khaja*, Henry James, Reginald P. Sequeira
}

\author{
Department of Pharmacology \\ and Therapeutics, College of \\ Medicine and Medical Sciences, \\ Arabian Gulf University, \\ Manama, Kingdom of Bahrain \\ Received: 09 February 2017 \\ Accepted: 07 March 2017 \\ *Correspondence to: \\ Dr. Khalid A. J. Al Khaja, \\ Email: khlidj@agu.edu.bh \\ Copyright: (C) the author(s), \\ publisher and licensee Medip \\ Academy. This is an open- \\ access article distributed under \\ the terms of the Creative \\ Commons Attribution Non- \\ Commercial License, which \\ permits unrestricted non- \\ commercial use, distribution, \\ and reproduction in any \\ medium, provided the original \\ work is properly cited.
}

\begin{abstract}
Background: Medical school training for students in pharmacotherapy is suboptimal and junior doctors are not confident to prescribe drugs. This study was conducted to assess the prescribing competency of students in preclerkship and clerkship phases at a medical school that implements problem-based learning curriculum.

Methods: Objective structured practical examination was used to assess prescribing competency of students in both phases. The prescribing performance was empirically categorized into poor, moderate and good competency.

Results: The prescription writing skills achieved by the clerkship students did not significantly differ from that attained by students in preclerkship phase. Good prescribing competency was attained by approximately $20 \%$ of the students at the end of both phases. Preclerkship students performed better on therapeutic reasoning than those in clerkship phase. Cognitive skill that involves choosing the superior $\operatorname{drug}(\mathrm{s})$ among several alternatives as achieved less satisfactorily by majority of students from both phases of the program.

Conclusions: Acquisition and further development of the prescribing competency during the clerkship phase was sub-optimal. The continuation of pharmacotherapeutic context-learning program during clerkship phase is recommended.
\end{abstract}

Keywords: Antihypertensives, Bahrain, Context-learning pharmacotherapy program, Prescribing skill assessment, Prescribing competency, Therapeutic reasoning

\section{INTRODUCTION}

A prescription is 'a written order, which includes detailed instructions of what medicine should be given to whom, in what formulation and dose, by what route, when, how frequently, and for how long. ${ }^{1}$ Ordering a valid and complete prescription requires a thorough knowledge and understanding of patho-physiology of disease, the pharmacological properties of the relevant drugs, and the ways in which the two dovetail. ${ }^{2}$

The drug-related components of a prescription include both inscription (i.e. appropriateness of $\operatorname{drug}(\mathrm{s})$ selected, strength, dosage form, quantity to be dispensed) and signtura (i.e. direction for use). The inscription and signtura are deemed to be the most challenging part of the prescription writing skill to be achieved by preclerkship medical students in both problem-based learning curriculum and traditional curriculum. ${ }^{3-6}$ Acquisition of therapeutic reasoning and prescribing competency, as a clinical skill, has been reported to be sub-optimal among students in preclerkship phase and junior doctors, regardless of the curriculum strategy used in medical schools. ${ }^{3-10}$

At the College of Medicine and Medical Sciences (CMMS), Arabian Gulf University (AGU), we have introduced a pharmacotherapeutic context learning program in order to develop the prescription writing competency of medical students at the preclerkship phase through a total of 16-hour educational interactive sessions. The program is interrupted thereafter during clerkship phase, assuming that the students' prescription writing competency would be further enhanced at realworld prescribing setting. 
The aim of this preliminary study is to propose a theoretical framework for assessing prescribing skill of medical students. In this study we have compared the prescribing competency of medical students (therapeutic reasoning and prescribing skill) at the end of preclerkship phase and those at the end of clerkship phase, respectively.

\section{METHODS}

The general methodology used in this study has been described by us previously as part of the ongoing curriculum evaluation by the department. ${ }^{3,4}$

\section{Study protocol}

“Approximately 150-200 students from Gulf Cooperation Council countries (Bahrain, Kuwait, Oman, Qatar, Saudi Arabia and United Arab Emirates) and other Arab countries are enrolled in medical program at the CMMS each year".

\section{Table 1: Medical curriculum map of the Arabian Gulf University.}

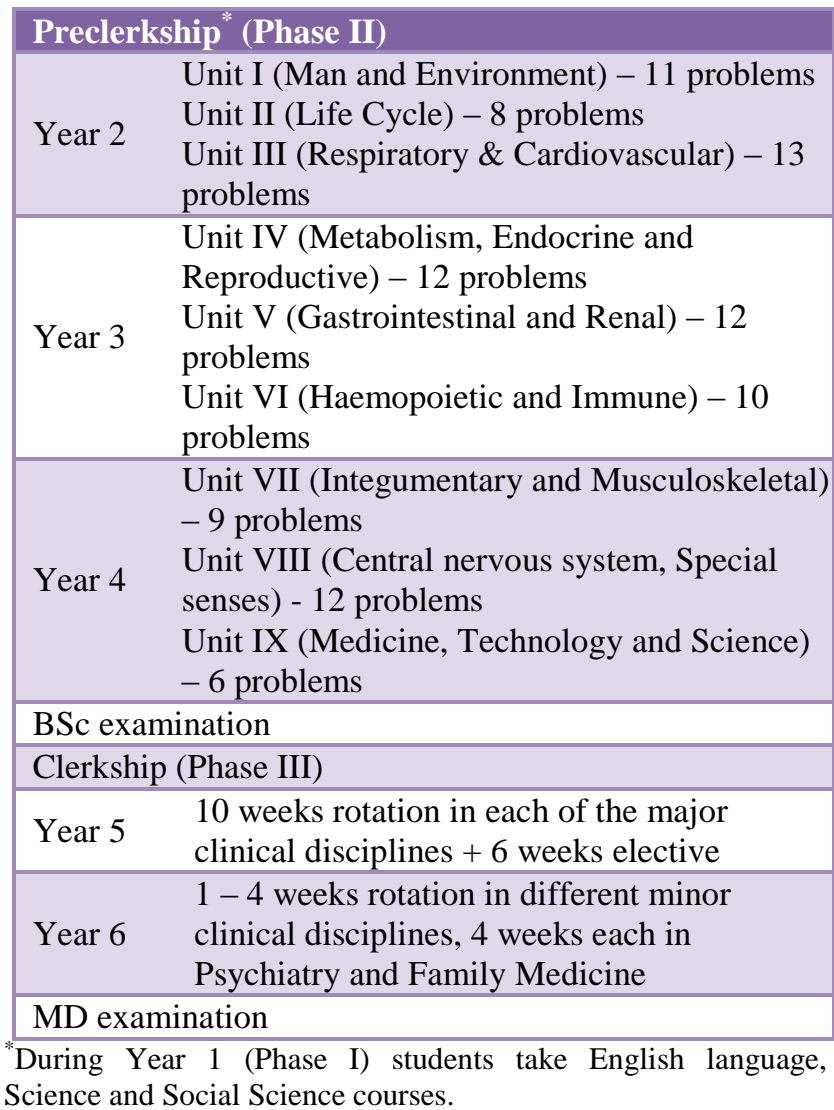

"The CMMS of the AGU, Bahrain, since its inception in early 1980s, adopted an integrated, problem-based, student-centered curriculum". The medical curriculum organization is shown in Table 1. "The medical program is divided into three phases: a premedical phase (1 year), a unit phase or preclerkship phase (3 years) and a clerkship phase (2 years). ${ }^{4}$ The preclerkship phase comprises 93 clinical problems and approximately 200 mini-problems. ${ }^{3}$ In this program that emphasizes selfdirected learning approach, basic and clinical knowledge, skills, ethics, values and attitudes are learned through health problems".

A context-learning pharmacotherapy program (medical treatment by means of drugs in a learning setting similar to the future professional setting) was introduced in the preclerkship phase in January 1995, by the Department of Pharmacology and Therapeutics. "At the end of each unit, the preclerkship medical students learn prescription order writing through an optional two-hour interactive session. Five to seven unit-related clinicotherapeutic case scenarios are given to the students as carry-home exercises to help them acquire critical appraisal skills, use of the drug formulary, and prescribing skills, particularly pre-compounded preparations for outpatient prescriptions and inpatient chart orders. These prescriptions are checked and formative feedback is provided. Thus, by the end of preclerkship phase, the students: (a) would have studied several clinicotherapeutic exercises integrating basic concepts and therapeutic principles related to common health problems given over a total of 16-hours interactive sessions; and (b) would be expected to be able to understand the therapeutic reasoning of drug prescribing and write valid prescriptions".

"The unit-related basic and clinical pharmacology and therapeutics-related learning objectives are tested at the end of each unit with comprehensive written examinations that include A-type MCQs, integrated cluster MCQs and multidisciplinary integrated short answer test items. The prescribing skills are tested through an end-unit objective structured practical examination (OSPE), 2-3 weeks post-intervention, by academic staff of the Department of Pharmacology \& Therapeutics".

"Based on the prescription format described, we divided the essential prescription components into physicianrelated and drug-related components. ${ }^{12}$ The physicianrelated components (P-RCs) include students' awareness of the necessity of writing (a) prescriber's name and professional degree; (b) date of prescription; (c) patient's identity and address; (d) the symbol $\mathrm{R}_{\mathrm{x}}$ Take Thou; (e) prescriber's signature; and (f) refill information. Each correctly written component was given a score of 6.25 out of 100. These components accounted for $37.5 \%$ of total score. The drug-related components (D-RCs) include (a) appropriateness of $\operatorname{drug}(\mathrm{s})$ selected; (b) strength of $\operatorname{drug}(\mathrm{s})$; (c) dosage form; (d) quantity to be dispensed; and (e) direction for use. Each correctly written component was given a score of 12.5 marks out of 100 . These components accounted for $62.5 \%$ of the total score. If any component was not written or incorrectly written, it was scored zero. In order to minimize inter-rater variations, a standard checklist was used to assess the prescribing skill performance in OSPE 
stations by two faculty members of the Department of Pharmacology \& Therapeutics. Each end-of-unit OSPE examination comprised of 25-35 stations representing the skills component of different disciplines. In each unit at least one OSPE station comprised of a 6-minute prescription writing exercise for a given clinical scenario. During the OSPE/ objective structured clinical examination (OSCE) the students were allowed access to the British National Formulary (BNF)".

\section{Tested clinical scenario}

During June 2012 a clinical scenario mentioned below was tested as one of the OSCE stations for the students at the end of clerkship phase (MD examinees) and as one of the OSPE stations for the students at the end of preclerkship (BSc examinees). In order to increase the sample size, in June 2013 the same clinical scenario was tested as an OSCE station for MD examinees.

Clinical scenario: During a routine health check-up at the primary health care center, a 53-year-old woman was found to have supine blood pressure of $148 / 92 \mathrm{mmHg}$, on multiple occasions. Laboratory investigation showed:

- Fasting blood glucose $5.8 \mathrm{mmol} / \mathrm{L}$ (reference range 3.3-5.8)

- Total cholesterol $5.1 \mathrm{mmol} / \mathrm{L}$ (reference range 5-6)

- LDL cholesterol $2.8 \mathrm{mmol} / \mathrm{L}$ (reference range 2-3)

- Triglyceride $1.7 \mathrm{mmol} / \mathrm{L}$ (reference range 0.2-1.8)

- Serum creatinine $82 \mu \mathrm{mol} / \mathrm{L}$ (reference range $60-90$ )

- Serum uric acid 480 $\mu \mathrm{mol} / \mathrm{L}$ (reference range 160-430)

The students were asked to write a complete prescription for managing hypertension in this patient.

\section{Operational definitions}

In this study, BSc examinees is an interchangeable term for students at the end of preclerkship phase, whereas MD examinees is an interchangeable term for students at the end of clerkship phase. "Absence of any P-RCs is deemed as minor errors of omission. Absence, vague, incomplete and/or illegibility of any component of D$\mathrm{RCs}$ is considered as major error of omission. Incorrectly written components of D-RCs are considered as an error of commission". ${ }^{13}$

The step of clinical reasoning pertaining to the choice of therapy is defined as therapeutic reasoning (therapeutic decision making). ${ }^{14}$ Good prescribing competency is defined as a situation when students demonstrate therapeutic reasoning skill and ability to write complete prescriptions without errors (or with only some minor errors of omission pertaining to P-RCs) i.e. having the skill of integration of knowledge (knows) into a rational and valid prescription (competence). Moderate prescribing competency is defined as a situation when students possess the therapeutic reasoning skill but not fully competent to write complete prescriptions as a result of several minor and major errors of omission, and/commission error(s) related to both P-RCs and DRCs. Poor prescribing competency is defined as a situation when students failed to demonstrate therapeutic reasoning and were unable to integrate knowledge (if any) into a valid prescription. Context-learning is defined as learning in a setting that is similar to the setting of the future professional. ${ }^{15}$

\section{Statistical analysis}

The statistical package of social sciences (SPSS/PC) version 19 was used for data entry and analysis. Chisquare test was used to test the differences between proportions and two-tailed student's t-test was used for continuous variables. A p-value less than 0.05 were considered statistically significant.

\section{RESULTS}

The mean total score of the combined P-RCs and D-RCs achieved by students at the end of preclerkship (BSc examinees) and clerkship (MD examinees) phase is presented in Table 2. Apart from prescriber's identity, appropriateness of $\operatorname{drug}(\mathrm{s})$ selection and patients' information, the total mean score of the prescribing writing skill attained by clerkship students did not significantly differ from that achieved by preclerkship students $(53.6 \pm 26.9$ vs $57.9 \pm 28.4 ; \mathrm{p}=0.173$; Table 2$)$.

Based on our scoring system, the students from both phases were categorized into those who demonstrated poor $(<60 \%$ score), moderate $(\geq 60 \%$ to $<87.5 \%$ score $)$ and good ( $\geq 87.5 \%$ score) prescribing competency (Table 3 ). The overall proportion of students who achieved poor, moderate and good prescribing competency was approximately $20 \%, 20-30 \%$, and $50-60 \%$, respectively.

It is evident that only one out of five students was competent to write a complete and valid prescription. The performance of antihypertensive therapeutic reasoning (therapeutic decision making) of medical students at the end of preclerkship and clerkship phases is shown in Table 4. Rational antihypertensives were more often prescribed by preclerkship $(61.1 \%)$ as compared to clerkship (45.5\%) students ( $\mathrm{p}=0.006$; Tables 2 and 4). Moreover, students in clerkship phase showed a greater tendency to prescribe irrational B-blockers and diuretics (at doses $>12.5 \mathrm{mg} /$ day) than preclerkship ones (28.1\% vs. $17.6 \% ; \mathrm{p}=0.027$; Table 4).

Among rational antihypertensives, angiotensin converting enzyme inhibitors (ACEIs) were the most commonly prescribed by preclerkship $[87.4 \%$ (118/135) $]$ as compared to clerkship [89.1\% (49/55)] students (Table 4). Among ACEIs, captopril was often chosen by students in preclerkship [73.7\% (87/118)] and clerkship $[87.7 \%(43 / 49)]$ phases $(\mathrm{p}=0.064)$. 
Table 2: Proportion of correctly written physician-related and drug-related components of prescriptions: B.Sc. examinees versus MD examinees.

\begin{tabular}{|c|c|c|c|}
\hline \multirow{2}{*}{ Students' phase } & \multicolumn{2}{|l|}{ Medical students } & \multirow{2}{*}{ p-Value } \\
\hline & B.Sc. ${ }^{a}$ examinees (Pre-clerkship) & MD ${ }^{\mathrm{b}}$ examinees (clerkship) & \\
\hline Total number of students & 221 & 121 & \\
\hline \multicolumn{4}{|l|}{ Physician-related components: $\mathrm{n}(\%)$} \\
\hline Prescriber's identity & $151(68.3)$ & $69(57.0)$ & $0.044^{\mathrm{c}}$ \\
\hline Date of prescription & $181(81.9)$ & $104(86.0)$ & 0.366 \\
\hline Patient's information & $143(64.7)$ & $110(90.9)$ & $0.0001^{\mathrm{c}}$ \\
\hline $\mathrm{Rx}$ (take thou) & $215(97.3)$ & $121(100)$ & 0.093 \\
\hline Prescriber's signature & $215(97.3)$ & $121(100)$ & 0.093 \\
\hline Refill information & $160(72.4)$ & $85(70.2)$ & 0.707 \\
\hline \multicolumn{4}{|l|}{ Drug-related components: n (\%) } \\
\hline Appropriateness of drug(s) selected & $135(61.1)^{d}$ & $55(45.5)^{d}$ & $0.006^{\mathrm{c}}$ \\
\hline Strength of drug & $116(52.5)$ & $52(43.0)$ & 0.113 \\
\hline Dosage form & $88(39.8)$ & 48 (39.7) & 1.000 \\
\hline Quantity to be dispensed & $65(29.4)$ & $28(23.1)$ & 0.252 \\
\hline Directions for use (sigma) & $88(39.8)$ & $40(33.0)$ & 0.243 \\
\hline Percentage of score (Mean \pm SD) & $57.9 \pm 28.4$ & $53.6 \pm 26.9$ & 0.173 \\
\hline
\end{tabular}

Table 3: Comparison between B.Sc. examinees and MD examinees pertaining to prescribing competency (knows how) based on Miller's pyramid components.

\begin{tabular}{|c|c|c|c|c|c|}
\hline & \multicolumn{4}{|c|}{ B.Sc. ${ }^{a}$ examinees Pre-clerkship $\quad$ MD $^{\mathrm{b}}$ examinees clerkship } & \multirow{2}{*}{ p-Value } \\
\hline & $\mathbf{N}(\%)$ & Mean score \pm SD & $\mathbf{N}(\%)$ & Mean score \pm SD & \\
\hline Total number of students & 221 & & 121 & & \\
\hline $\begin{array}{l}\text { Students who demonstrated poor } \\
\text { prescribing competency c }(<60 \text { score })\end{array}$ & $111(50.2)$ & $32.3 \pm 11.9$ & $72(59.5)$ & $33.1 \pm 8.1$ & 0.112 \\
\hline $\begin{array}{l}\text { Students who demonstrated moderate } \\
\text { prescribing competencyc }(>60 \text { to }<87.5 \\
\text { score) }\end{array}$ & $67(30.3)$ & $75.9 \pm 7.7$ & $26(21.5)$ & $73.1 \pm 7.5$ & 0.098 \\
\hline $\begin{array}{l}\text { Students who demonstrated }{ }^{\mathrm{c}} \text { good } \\
\text { prescribing competency (>87.5 to100 } \\
\text { score) }\end{array}$ & $43(19.5)$ & $95.8 \pm 5.1$ & 23(19.0) & $95.4 \pm 4.7$ & 1.000 \\
\hline
\end{tabular}

The overall prescribing of antihypertensive combination therapies (two-drug combination and fixed-dose combinations) was $3.2 \%(7 / 221)$ and $7.4 \%$ (9/121) for students at preclerkship and clerkship phases, respectively. The rate of prescribed complementary combination therapy, such as ACEI + diuretic, was $2.3 \%$ $(5 / 221)$ for preclerkship and $2.5 \%$ (3/121) for clerkship students.

\section{DISCUSSION}

\section{Prescribing skill assessment}

This study provides an insight into prescribing skill of medical students in preclerkship and clerkship phases in a problem-based learning program. The total mean score of the P-RCs and D-RCs attained by preclerkship students was sub-optimal and almost comparable to previous studies conducted at the same setting using the same context-learning pharmacotherapy program. ${ }^{3,4}$ Apart from prescriber's identity, appropriateness of drug(s) selection and patients' information, the prescribing skill achieved by clerkship students did not significantly differ from that achieved by preclerkship students (Table 2). These findings contrast those reported by others. ${ }^{16,17}$ Richir et al reported that a preclinical context-learning pharmacotherapy program leads to the use of more rational prescribing modalities by medical students during clerkship phase. ${ }^{16}$ Vollebregt et al demonstrated that the therapeutic reasoning, an important step in a clinical reasoning for the choice of therapy, achieved by 
preclinical students who had followed a context-learning pharmacotherapy program was significantly inferior than that by the final year medical students who had not followed the preclinical pharmacotherapeutics context learning program; however, the skill for obtaining patient information was better in preclinical students. ${ }^{17}$

Table 4: Antihypertensive agents prescribed by B. Sc. examinees and MD examinees.

\begin{tabular}{|c|c|c|}
\hline Phase of medical program & B. Sc. ${ }^{a}$ examinees $\mathbf{n}(\%)$ & MD $^{b}$ examinees $\mathbf{n}(\%)$ \\
\hline Total number of students & 221 & 121 \\
\hline \multicolumn{3}{|l|}{ Rational Antihypertensives } \\
\hline Angiotensin converting enzyme inhibitors & $118(53.4)^{\mathrm{c}}$ & $49(40.5)$ \\
\hline Angiotensin II receptor blockers & $6(2.7)$ & $1(0.8)$ \\
\hline Calcium channel blockers CCBs (DHP) & $6(2.7)$ & $2(1.7)$ \\
\hline Two-drug combinations ${ }^{d}$ & $4(1.8)$ & $3(2.5)$ \\
\hline A fixed dose combination $\left(\text { Co-zidocapt }{ }^{\circledR}\right)^{e}$ & $1(0.5)$ & - \\
\hline \multicolumn{3}{|l|}{ Non-rational antihypertensives } \\
\hline Diuretics $^{\mathrm{f}}$ & $16(7.2)^{\mathrm{c}}$ & $18(14.9)$ \\
\hline$\beta$-blockers [negative effects on metabolic syndrome] & $23(10.4)$ & $16(13.2)$ \\
\hline Methyldopa [restricted for hypertension in pregnancy] & $6(2.7)$ & $1(0.8)$ \\
\hline Clonidine [causes hypertensive crisis on sudden withdrawal] & $1(0.5)$ & - \\
\hline $\begin{array}{l}\text { Diazoxide [vasodilator given intravenously in hypertensive } \\
\text { emergency] }\end{array}$ & $1(0.5)$ & - \\
\hline $\begin{array}{l}\text { Hydralazine [vasodilator used for hypertensive crisis including } \\
\text { pregnancy] }\end{array}$ & $9(4.1)$ & $9(7.4)$ \\
\hline Minoxidil [vasodilator used for resistant hypertension] & $1(0.5)$ & - \\
\hline $\begin{array}{l}\text { Terazosin and indoramin [ } \alpha \text {-blockers used as add-on therapy in } \\
\text { treatment of resistant hypertension and BPH] }\end{array}$ & $4(1.8)$ & $4(3.3)$ \\
\hline $\begin{array}{l}\text { Diltiazem [CCB used for treatment and prophylaxis of angina } \\
\text { pectoris] }\end{array}$ & $3(1.4)$ & - \\
\hline Verapamil [CCB used for ventricular arrhythmias] & $6(2.7)$ & - \\
\hline $\begin{array}{l}\text { Spironolactone }\left[\mathrm{K}^{+} \text {sparing diuretic used for }\right. \\
\text { hyperaldosteronism] }\end{array}$ & $2(0.9)$ & $5(4.1)$ \\
\hline $\begin{array}{l}\text { Sildenafil [phosphodiesterase- } 5 \text { inhibitor for pulmonary } \\
\text { hypertension] }\end{array}$ & $1(0.5)$ & - \\
\hline Iloprost [vasodilator for primary pulmonary hypertension] & $2(0.9)$ & - \\
\hline Allopurinol [uric acid lowering agent] & $1(0.5)$ & $4(3.3)$ \\
\hline Nitrazepam [benzodiazepine used as sedative-hypnotic] & $1(0.5)$ & - \\
\hline Co-amilofruse ${ }^{\mathrm{g}}$ [fixed dose combination] & - & $1(0.8)$ \\
\hline Antihypertensive two-drug combination & $2(0.9)$ & $5(4.1)$ \\
\hline Non-specified ACE inhibitors & $1(0.5)$ & - \\
\hline Non-specified diuretics & $4(1.8)$ & $3(2.5)$ \\
\hline No medication prescribed & $1(0.5)$ & - \\
\hline Total & $221(99.9)$ & $121(99.9)$ \\
\hline
\end{tabular}

${ }^{\mathrm{a}}$ Bachelor of Basic Medical Sciences (BSc) degree is awarded at the end of phase II medical program (pre-clerkship program); ${ }^{b}$ Doctor of Medicine (MD) degree is awarded at the end of phase III clerkship program; ${ }^{c} \mathrm{p}<0.05 ;{ }^{\mathrm{d}}$ an ACE + a diuretic combination; ehydrochlorothiazide $12.5 \mathrm{mg} /$ captopril $25 \mathrm{mg}$, ${ }^{\mathrm{f}}$ diuretics at high doses $(>12.5 \mathrm{mg} / \mathrm{d})$; ${ }^{\mathrm{g}}$ amiloride $2.5 \mathrm{mg} / \mathrm{furosemide} 20 \mathrm{mg}$; BPH, benign prostatic hypertrophy; CCB, calcium channel blocker, DHP, dihydropyridine

A plausible explanation for sub-optimal development and acquisition of prescribing competency during the clerkship phase may be related to several confounding factors, possibly inadequate monitoring of students by mentors. Interruption of context-learning pharmacotherapy program during clerkship phase (Year 5 \& 6) may result in attrition and regression of the prescribing competency gained previously during the preclerkship phase has to be considered. The prescribing competency of medical students in clerkship program has been reported to be improved by twice-monthly rational prescribing session provided during the required internal medicine rotation for Year 4 medical students. ${ }^{18}$ Differences in students' intrinsic motivation and academic strength could have influenced such skills. Suboptimal student attendance in pharmacotherapeutic context-learning program has been reported to be a significant predictor of performance in prescribing skill. ${ }^{4}$ 
To what extent the above-mentioned confounding variables would have influenced the prescribing competency of students in clerkship phase is debatable. A further study to evaluate the impact of pharmacotherapeutic context learning intervention (whether voluntary or mandatory) on the prescribing competency of students at clerkship phase is needed.

Our findings were in line with those reported by Celebi et al who demonstrated that five or more weeks of clerkship in internal medicine did not reduce the students' prescription related errors as compared to those committed by other students before commencing their clerkship in the same department. ${ }^{19}$ These authors suggested that specific prescription training program is warranted to prevent the drug related problems.

Assessment in medical education is no longer based on knowledge testing because knowledge alone is insufficient to predict clinical performance in practice. ${ }^{20}$ Miller proposed a pyramid model which conceptualizes the essential facets of clinical assessment. ${ }^{20}$ The base of pyramid represents the knowledge component of competence- knows and knows how. Knows indicates that a student, a resident, a physician must know what is required in order to carry out those professional functions effectively (knowledge). In knows how component, graduates must develop the skill of acquiring information from a variety of human and laboratory sources, to analyze and interpret these data, and finally to integrate such findings into a rational diagnostic or management plan (competence). In shows how, the students must not only be able to demonstrate that they know and know how, but also shows how they do it when faced with a patient (performance). The does, the apex component of the pyramid focuses on what a graduate actually does when functioning independently in clinical practice.

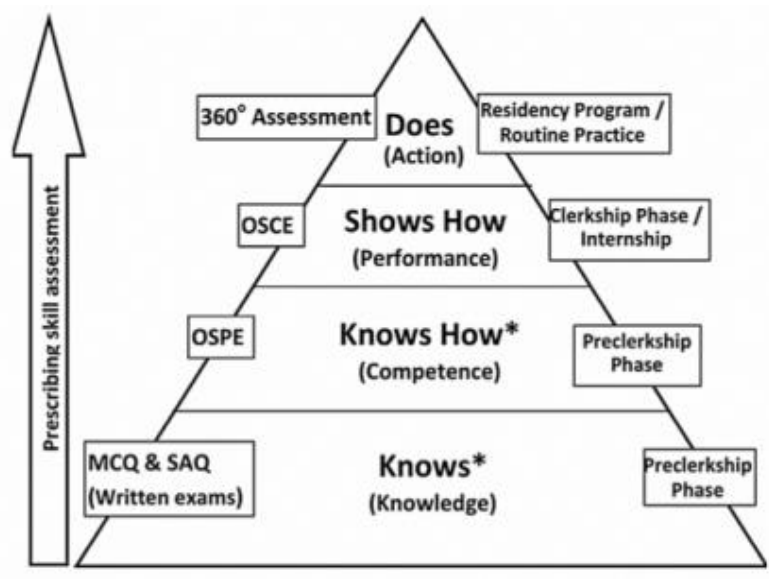

Figure 1: Conceptual model that links Miller's pyramid approach for prescribing skill assessment.

The fundamental cognitive principle that underpins prescribing skill is that a) knowledge (knows) is a prerequisite for the interpretation (knows how), and b) both knowledge and interpretation are essential for problem solving (shows/shows how/does). Thus knows, knows how, shows, shows how and does should be considered as a continuum, and prescribing skill incorporates these dimensions at different stages of the medical training until mastery is achieved (Figure 1).

At the CMMS, AGU we follow a conceptual teaching and evaluation model derived from Miller's pyramid with slight modifications on evaluating strategies (Figure 1). Assessment of medical graduates has tended to focus mainly on Miller's pyramid components knows and knows how in early stages of medical curriculum. ${ }^{21}$

Based on the levels of prescribing competency which mainly depend on students' knows and knows how assessment and on our scoring, sub-analysis of our data revealed that at the end of preclerkship and clerkship phases students have demonstrated poor, moderate and good prescribing competencies (Table 3). With respect to attainment of a good prescribing competency (a complete and rational prescription), no significant difference was evident between students of both phases. This important professional skill was adequately achieved by approximately $20 \%$ of preclerkship and clerkship students- i.e. only one out of five students was competent to write a legible, unambiguous and complete prescription which meets the professional requirements. Despite their awareness about the principle of therapeutic reasoning, approximately $20-30 \%$ of the students from both phases were not fully competent to write complete prescriptions: several minor or major errors of omission and / or commission error(s) pertaining to P-RCs and DRCs were committed (moderate prescribing competency). Unfortunately majority of the students (50-60\%) have demonstrated poor prescribing competency as a result of a lack of therapeutic reasoning and / or inability to integrate knowledge, if any, into rational prescriptions (Table 3).

Notwithstanding the reliability, our findings suggest that students did not acquire and develop adequate prescribing competency during the clerkships phase. In order to improve students prescribing skills, we propose that the pharmacotherapeutics context learning program has to be mandatory in clerkship phase by providing several sessions in pharmacotherapeutics for students. Moreover, mentorship behaviors and mentorship quality with feedback should be considered to maximize effectiveness.

\section{The therapeutic reasoning skill assessment}

Therapeutic reasoning (therapeutic decision making) is defined as an important part of clinical reasoning process that pertains to the choice of therapy of a clinical problem in a specific patient. ${ }^{14}$ Based on the clinical scenario tested in OSPE station, a monotherapy with either ACEIs, angiotensin II receptor blockers (ARBs) and dihydropyridine calcium channel blockers (CCBs) or their combination therapy with a low dose of thiazide and 
thiazide-like diuretics ( $\leq 12.5 \mathrm{mg} /$ day) was deemed to be the recommended therapy of choice. This is because these antihypertensives induce a neutral or positive effect on glycemic control, serum lipid profile and uric acid level. Contrary to expectations, we found that the therapeutic decision making process was better achieved by preclerkship students as compared to clerkship students (Tables 2 and 4). Moreover, this finding was again re-affirmed by overt prescribing of antihypertensives such as B-blockers and diuretics, that are deemed to be less optimal drugs for the tested OSPE scenario, by clerkship students as compared to preclerkship students $(28.1 \%$ vs $17.6 \%$; $=0.027$; Table 4). Of note, ß-blocker based initial therapy for hypertension is not preferred. $^{22,23}$ Unlike nebivolol and carvedilol, the third generation $\beta$-blockers, other $\beta$ blockers, in particular atenolol, cause adverse effects on the components of the cardio-metabolic syndrome such as lipid profile, insulin sensitivity and increase the incidence of new-onset diabetes. ${ }^{24-28}$ Thiazide and thiazide-like diuretics (at doses $\geq 25 \mathrm{mg} /$ day) are associated with hyperglycemia, hyperuricemia, hyperlipidemia, hypokalemia (which may contribute to cardiac arrhythmias) and other electrolyte changes. ${ }^{22,29}$ Long-term use of diuretics can also increase the cost of treatment owing to the therapeutic interventions needed to treat diuretic-induced metabolic and electrolyte abnormalities. ${ }^{25,30}$ In addition to prescribing B-blockers and diuretics several irrational drug therapies have been chosen by both preclerkship $(21.3 \%)$ and clerkship $(26.4 \%)$ medical students such as antihypertensives those reserved for treatment of severe hypertension resistant to other drugs, those indicated for treatment of pulmonary hypertension, pregnancy induced hypertension and those for which antihypertensive therapeutic use has been limited owing to their adverse effects (Table 4).

Based on the tested clinical scenario, ACEIs as one of the favored antihypertensive class was the most often prescribed class by preclerkship and clerkship phase students (Table 4). Among ACEIs, there was a significant preference for captopril by both preclerkship and clerkship students although captopril is not the best option as compared with other ACEIs. Captopril has a short half-life and should therefore be administered in multiple daily dosing to achieve effective blood pressure control. In contrast, long-acting ACEIs notably lisinopril, perindopril, fosinopril etc have superior pharmacokinetic profiles and these can be administered once daily to improve compliance to drug therapy and to reduce blood pressure variations. ${ }^{31}$ This finding may suggest that the pharmacotherapeutic reasoning involved in choosing the rational antihypertensive(s), among several alternatives, is not of highest standard or quality for majority of students from both preclerkship and clerkship phases.

\section{CONCLUSION}

The best of our knowledge, this study is deemed to be the first to evaluate the differences in prescribing competencies attained by students in preclerkship and clerkship students in problem-based medical curriculum. The prescribing competency further achieved by students after clerkship phase did not significantly differ from that attained by students at the end of preclerkship phase. Good prescribing competency was apparent only in approximately $20 \%$ of students from both phases based on limited sampling. Preclerkship students demonstrated better therapeutic reasoning than those in clerkship phase. The pharmacotherapeutic reasoning that involves choosing the better antihypertensive(s) among several alternatives is not of highest standard or quality for students in both phases. Acquisition and further development of prescribing competency during the clerkship phase were sub-optimal. The impact of intervention with structured pharmacotherapeutic context learning during the clerkship phase on prescribing competency needs to be explored. The generalizability of our findings needs further validation using multiple clinical scenarios.

\section{ACKNOWLEDGMENTS}

Authors would like to acknowledge the cooperation of Prof. Akbar Mohsin Mohammed, coordinator of MD exam, and assistance given by Ms. Mini James in preparing this manuscript.

Funding: No funding sources Conflict of interest: None declared

Ethical approval: The study was approved by the Department of Pharmacology \& Therapeutics council for course evaluation

\section{REFERENCES}

1. Aronson JK. Medication errors: definitions and classification. Br J Clin Pharmacol. 2006;67:599-604.

2. Aronson JK. A prescription for better prescribing. $\mathrm{Br}$ J Clin Pharmacol. 2006;6:487-91.

3. Al Khaja KA, Handu SS, James H, Mathur VS, Sequeira RP. Assessing prescription writing skills of pre-clerkship medical students in a problem-based learning curriculum. Int $\mathrm{J}$ Clin Pharmacol Ther. 2005;43:429-35.

4. Al Khaja KAJ, James H, Sequeira RP. Effectiveness of an educational intervention on prescription writing skill of preclerkship medical students in a problembased curriculum. J Clin Pharmacol. 2013;53(5):48390.

5. Rauniar GP, Roy RK, Das BP, Bhandari G, Bhattacharya SK. Prescription writing skills of preclinical medical and dental undergraduate students. J Nepal Med Assoc. 2008;47:197-200.

6. Kumar J, Shaik MM, Kathi MC, Chetty MS, Deka A. Appraisal of prescription writing skills of preclinical undergraduate students in a medical institute of Nepal. JCMS-Nepal. 2010;6:7-13. 
7. Coombes ID, Stowasser DA, Coombes JA, Mitchell C. Why do interns make prescribing error? A quantitative study. Med J Aust. 2008;188:89-94.

8. Likic R, Maxwell SRJ. Prevention of medication errors: teaching and training. Br J Clin Pharmacol. 2009;67:656-61.

9. Dean B, Schachter M, Vincent C, Barber N. Prescribing errors in hospital inpatients: their incidence and clinical significance. Qual Saf Health Care. 2002;11:340-44.

10. Ross S, Bond C, Rothnie H, Thomas S, Macleod MJ. What is the scale of prescribing errors committed by junior doctors? A systematic review. $\mathrm{Br} \mathrm{J}$ Clin Pharmacol. 2009;67:629-40.

11. Mathur VS, Sequeira RP, Jassim KA. Promoting rational prescribing in problem-based curriculum. In: Scherpier AJJA, van der Vleuten CPM, Rethans RJ, van der Steig AFW, eds. Advances in Medical Education. Dordrecht: Kluwer Academic Publishers; 1997:516-18.

12. Lofholm PW, Katzung BG. Rational prescribing and prescription writing. In: Katzung BG, ed. Basic and Clinical Pharmacology. $11^{\text {th }}$ Ed. New York, NY: McGraw-Hill; 2009:1127-1139.

13. Al Khaja KAJ, Sequeira RP, Al-Ansari T, Damanhori AH, James H, Handu SS. Paediatric iron preparations for infants in Bahrain: Some therapeutic concerns. Int J Clin Pharmacol Ther. 2010;48:200-5.

14. Norman G. Research in Clinical Reasoning: Past history and current trends. Med Edu. 2005;39:418-27.

15. Richir MC, Tichelaar J, Geijteman ECT, de Vries TP. Teaching Clinical Pharmacology and therapeutics with emphasis on the therapeutic reasoning of undergraduate medical students. Eur J Clin Pharmacol. 2008;64:217-24.

16. Richir MC, Tichelaar J, Stanm F, Thijs A, Danner SA, Schneider AJ, et al. A context-learning pharmacotherapy program for preclinical medical students leads to more rational drug prescribing during their clinical clerkship in internal medicine. Clin Pharmacol Ther. 2008;84:513-6.

17. Vollebregt JA, Oldenrijk JV, Kox D, van Galen SR, Sturm B, Metz JC, et al. Evaluation of a pharmacotherapy context-learning program for preclinical medical students. Br J Clin Pharmacol. 2006;62:666-72.

18. Zgheib NK, Simaan JA, Sabra R. Using team-based learning to teach clinical pharmacology in medical school: Student satisfaction and improved performance. J Clin Pharmacol. 2011;51:1101-11.

19. Celebi N, Wagner R, Weyrich P, Heine D, Fenik Y, Holderried F, et al. Medical clerkships do not reduce common prescription errors among medical students. Naunyn Schmiedebergs Arch Pharmacol. 2010;382:171-6.
20. Miller GE. The assessment of clinical skills, competence, performance. Acad Med. 1990;65:63-7.

21. Wass V, Van der Vleuten C, Shatzer J, Jones R. Assessment of clinical competence. Lancet. 2001;357(9260):445-9.

22. National Institute for Health and Clinical Excellence (NICE) [homepage on internet]. Hypertension in adults: diagnosis and management. NICE guidelines [CG127]. Available from: https://www.nice.org.uk/guidance/cg127/chapter/1Guidance. Accessed 6 February 2017.

23. James PA, Oparil S, Carter BL, Cushman WC, Dennison-Himmelfarb C, Handler J, Lackland DT, et al. Evidence-based guidelines for the management of high blood pressure in adults Report from the panel members appointed to the eighth joint National Committee (JNC8). JAMA. 2014;311:507-20.

24. Tziomalos K, Athyros VG, Karagiannis A, Mikhailidis DP. Dyslipidemia induced by drugs used for the prevention and treatment of vascular diseases. Open cardiovasc Med J. 2011;5:85-9.

25. Deshmukh M, Lee HW, McFarlane SI, WhaleyConnell A. Antihypertensive medications and their effects on lipid metabolism. Curr Diab Rep. 2008;8:214-20.

26. Carella AM, Antonucci G, Conte M, Di Pumpo M, Giancola A, Antonucci E. Antihypertensive treatment with beta-blockers in the metabolic syndrome: a review. Curr Diabetes Rev. 2010;6:215-21.

27. Mancia G, Gassi G, Zanchetti A. A new onset diabetes and antihypertensive drugs. J Hypertens. 2006;24:3-10.

28. Opie LH, Schall R. Old antihypertensives and new diabetes. J Hypertens. 2004;22:1453-8.

29. European Heart Journal [homepage on internet]. 2013 $\mathrm{ESH} / \mathrm{ESC}$ Guidelines for the management of arterial hypertension. Available from: http://eurheartj.oxfordjournals.org/content/34/28/215 9. Accessed 6 February 2017.

30. Salvetti A, Chiadoni L. Thiazide diuretics in the treatment of hypertension: an update. J AM Soc Nephrol. 2006;17:S25-9.

31. World Health Organization, International Society of Hypertension Writing Group. World Health Organization (WHO) / International Society of Hypertension (ISH) Statement on management of hypertension. J Hypertens. 2003;21:983-92.

Cite this article as: Al Khaja KAJ, James $\mathrm{H}$, Sequeira RP. Acquisition of prescribing skill by medical students in a problem-based learning curriculum: preclerkship to clerkship phase transition. Int J Basic Clin Pharmacol 2017;6:718-25. 